\title{
Improved screening for colorectal cancer by immunological detection of occult blood
}

\author{
D J FROMMER, A KAPPARIS, M K BROWN
}

\begin{abstract}
A radial immunodiffusion technique for detecting faecal haemoglobin and the Hemoccult II kit used with and without rehydration of the faecal sample were compared in a screening programme for bowel cancer, in which 1328 subjects took part.

A positive result was obtained in 170 (13\%) subjects. Nineteen of the 153 subjects investigated were found to have colorectal carcinomas and 52 had polyps (40 with adenomas). Radial immunodiffusion and Hemoccult II with and without rehydration detected bleeding in, respectively, all 19,15 , and 11 subjects with colorectal carcinoma. Hemoccult II with and without rehydration detected only seven and six, respectively, of 11 Dukes's stage A carcinomas, whereas all 11 were detected with the immunological test. Hemoccult II with and without rehydration and radial immunodiffusion detected bleeding from adenomas in, respectively, 22, 14, and 34 of the $\mathbf{4 0}$ subjects. False positive results occurred in 55 out of 1302 subjects by Hemoccult II with rehydration, in 28 out of 1304 by Hemoccult II without rehydration, and in 50 out of 1304 by the immunological technique; true positive results were defined as bleeding from carcinomas and adenomas.

Immunological detection of occult blood in faecal samples seems to show more adenomas and carcinomas (particularly early lesions) than the Hemoccult II kit and has a rate of false positive results that is acceptably low.
\end{abstract}

\section{Introduction}

Colorectal cancer has become the most common form of internal cancer in many Western nations, but surgical, chemotherapeutic, and radiotherapeutic techniques have done little to improve mortality during the past two decades. An alternative strategy to reduce mortality is earlier diagnosis in the population-that is, screening of subjects without symptoms for early cancers. Early diagnosis by detection of faecal occult blood has had promising results and been increasingly accepted by the general public in the past decade.

Screening programmes have used chemical techniques for detecting faecal occult blood that are based on the pseudoperoxidase activity of haemoglobin on slides impregnated with guaiac. The most commonly used of the commercially available kits is Hemoccult II (Smith-Kline Diagnostics, Sunnyvale, California), which is simple, inexpensive, and rapid, a result being available in 30 seconds. An important disadvantage of such chemical techniques is that they are not specific for blood (human or animal), giving false positive results when red meat and fruit and vegetables containing peroxidase have been ingested. ${ }^{2}$ Dietary restrictions are therefore necessary when collecting faecal samples, which may reduce compliance in screening programmes. In addition, Hemoccult II (without faecal rehydration) detected $72 \%$ or less of symptomatic colorectal carcinomas, ${ }^{3.5}$ though rehydration of the faecal samples increased its sensitivity. ${ }^{45}$

Immunological techniques are more specific and sensitive than

Department of Medicine, St Vincent's Hospital, Darlinghurst, New South Wales 2010, Australia

D J FROMMER, MD, FRCP, senior lecturer

A KAPPARIS, BSC, scientific officer

M K BROWN, bowel cancer screening coordinator

Correspondence to: Dr Frommer. chemical ones. A radial immunodiffusion technique developed by this hospital's bowel cancer screening unit had a limit of sensitivity of $2 \mathrm{mg}$ haemoglobin/100 $\mathrm{g}$ faeces, ${ }^{6}$ whereas Hemoccult II had a limit of sensitivity of about $200 \mathrm{mg} / 100 \mathrm{~g}$ faeces. ${ }^{7}$ The rate of false negative results in patients with colorectal carcinomas was nearly zero with the immunological technique compared with $13-28 \%$ with Hemoccult II. ${ }^{5}$ We compared these chemical and immunological techniques in our screening programme to assess the value of the new radial immunodiffusion technique in the early detection of bowel cancers and premalignant polyps.

\section{Subjects and methods}

During 18 months 1328 subjects were screened for occult bleeding by the Hemoccult II kit and radial immunodiffusion. Subjects selected themselves through a publicly available cancer screening programme. Most of them were over 40 , with a mean age of 58.5 (SD 12.5) years. They were asked to adhere to a six day diet, which was free of red meat and fruit and vegetables containing peroxidase, for the duration of the test and to collect six faecal samples on filter paper for immunological testing. ${ }^{2}$ Faecal specimens for the Hemoccult II kits were collected on the last three days of the diet such that six faecal samples were available for testing by the unrehydrated and six by the rehydrated techniques (see below). Subjects who gave positive results by any of the three techniques were asked to have follow up investigations at this hospital, which consisted of taking a history and having a physical examination, rigid sigmoidoscopy, and colonoscopy. Some subjects, however, had a double contrast barium enema instead of or in addition to colonoscopy for various reasons-for example, unwillingness to undergo colonoscopy or inadequate visualisation of the colon.

The completed Hemoccult II and immunological kits were returned together and then processed as soon as possible to avoid any loss of detectable haemoglobin. Hemoccult II cards were developed on the day of receipt both with and without rehydration of faecal specimens. For rehydration one drop of water was added to the faecal smears 30 minutes before development, and these smears were then processed according to Hemoccult II's standard directions.

The immunological samples were developed with a radial immunodiffusion technique as described by Songster $e t a l^{8}$ and subsequently modified by us. ${ }^{5}$ Faeces smeared on to filter paper showed a fall in detectable haemoglobin concentrations of $1 \cdot 34 \%$ a day. ${ }^{6}$ Three millimetre discs of faeces on filter paper were punched out and placed in wells in an agarose gel plate containing a 5\% titre of rabbit immunoglobulin to human haemoglobin (Dakopatts, Denmark). Precipitin rings developed overnight at room temperature and were stained. The rings' diameters were measured and concentrations calculated from a range of haemoglobin standards. The limit of detection of this system is about $2.0 \mathrm{mg} / 100 \mathrm{~g}$ faeces with a coefficient of variation of $5.9 \%$ at $250 \mathrm{mg} / 100 \mathrm{~g}$ faeces. ${ }^{5}$ This technique gave negative results when tested with blood or meat from cattle, sheep, pigs, and chicken.

Statistical values were obtained with McNemar's test with continuity correction $^{9}$ and were used to compare sensitivities and specificities of the three techniques. Means and standard deviations are given.

\section{Results}

Screening for bowel cancer by a radial immunodiffusion technique and Hemoccult II with and without rehydration resulted in $170(13 \%)$ subjects

TABLE I-Distribution of positive results obtained in screening programme by Hemoccult II and immunological techniques. Values are proportions (percentages)

\begin{tabular}{|c|c|c|c|}
\hline & \multicolumn{2}{|c|}{ Hemoccult II } & \multirow{2}{*}{$\begin{array}{l}\text { Immunological } \\
\text { technique }\end{array}$} \\
\hline & Not rehydrated & Rehydrated & \\
\hline $\begin{array}{l}\text { Subjects } \\
\text { Samples }\end{array}$ & $\begin{array}{c}58 / 169(34) \\
150 / 1012(15)\end{array}$ & $\begin{array}{l}108 / 167(65) \\
340 / 1000(34)\end{array}$ & $\begin{array}{l}121 / 170(71) \\
311 / 1017(31)\end{array}$ \\
\hline
\end{tabular}


having positive results by at least one method. Table I shows the results obtained. Follow up investigations were refused by 17 subjects. In five subjects the investigations were incomplete as neither colonoscopy nor barium enema was carried out for various reasons. Colorectal carcinoma was diagnosed in 19 patients and adenoma in 40 , comprising $1.4 \%$ and $3.0 \%$ of the population screened, respectively. Although 82 subjects had neither adenoma nor carcinoma, other possible causes of bleeding were identified in 30: metaplastic polyps (five), polyps of unknown histological type (seven), diverticulitis (eight), telangiectases (five), haemorrhoids (three), and colitis (two). No abnormality was found in 59 patients. Haemorrhoids and diverticula were accepted as causes of bleeding only when there was clinical or other confirmatory evidence.

\section{CARCINOMAS}

The mean age of subjects with carcinoma was $65 \cdot 5(9 \cdot 3)$ years. Radial immunodiffusion detected bleeding in all 19 subjects with colorectal carcinoma, whereas Hemoccult II with and without rehydration detected it in only 15 and 11 , respectively ( $p>0.05$ and $p<0.025$ compared with the immunological technique) (table II). The colorectal carcinomas were classified according to Dukes's stages with the addition of a metastatic stage, D (table III). Hemoccult II with and without rehydration detected bleeding in $64 \%$ and $55 \%$ respectively of the stage $\mathrm{A}$ carcinomas detected with the immunological technique. The specificity of both the immunological and the Hemoccult II rehydrated techniques was less than that of the Hemoccult II technique without rehydration ( $p<0.025$ and $p<0.0005$, respectively).

The haemoglobin concentrations obtained by radial immunodiffusion for the subjects with carcinoma ranged from $2 \cdot 1$ to $2630 \mathrm{mg}$ haemoglobin $/ 100 \mathrm{~g}$ faeces. Almost half of the samples that gave positive results by radial immunodiffusion had concentrations well below the limit of detection of Hemoccult II (about $200 \mathrm{mg}$ haemoglobin/100 g faeces.) No correlation seemed to exist between a positive stool sample by any technique and site of carcinoma, except that carcinomas in the sigmoid and descending colons seemed to bleed less frequently.

\section{ADENOMAS}

The radial immunodiffusion technique and Hemoccult II with and without rehydration detected bleeding in 40,29 , and 16 , respectively, of the 52 subjects with polyps. These 52 subjects had 90 polyps, of which 60 were adenomas (six with dysplasia). The mean age of subjects with adenomas was $61.5(11 \cdot 4)$ years. The 60 adenomas were in 40 subjects, and occult blood was detected by the immunological technique and Hemoccult II with and without rehydration in 34,22 , and 14 , respectively (table IV).

Among 82 subjects without adenoma or carcinoma a positive result was obtained in $50(61 \%), 55(67 \%)$, and $28(34 \%)$ by radial immunodiffusion and Hemoccult II with and without rehydration, respectively, which is equivalent to $4 \%, 4 \%$, and $2 \%$ of the population screened. False positive results were determined in subjects who had colonoscopy or barium enema, or both, being found not to have carcinoma or adenoma (table V). Thus subjects with other causes of bleeding-for example, metaplastic polyps, colitis, and diverticulitis-were regarded as yielding false positive results.

Table VI shows that the positive predictive value-that is, the ratio of true positive to total positive results - of all three techniques tended to increase with the number of positive samples. The predictive value of a positive result

TABLE II-Detection of carcinoma in screening programme by Hemoccult II and immunological techniques. Values are numbers (percentages)

\begin{tabular}{lccc}
\hline & \multicolumn{2}{c}{ Hemoccult II } & Immunological \\
\cline { 2 - 3 } & Not rehydrated & Rehydrated & $\begin{array}{c}\text { Imnnique } \\
\text { tech }\end{array}$ \\
\hline Subjects $(n=19)$ & $11(58)$ & $15(79)$ & $19(100)$ \\
Samples $(n=114)$ & $46(40)$ & $65(57)$ & $79(69)$ \\
\hline
\end{tabular}

TABLE III-Numbers of subjects with carcinomas according to Dukes's staging detected in screening programme by Hemoccult II and immunological techniques.

\begin{tabular}{lccc}
\hline & \multicolumn{2}{c}{ Hemoccult II } & Immunological \\
\cline { 2 - 3 } Dukes's stage & Not rehydrated & Rehydrated & $\begin{array}{c}\text { Imique } \\
\text { techni }\end{array}$ \\
\hline A $(n=11)$ & 6 & 7 & 4 \\
B $(n=4)$ & 3 & 4 & 3 \\
C $(n=3)$ & 2 & 3 & 1 \\
D $(n=1)$ & 1 & 1 & \\
\hline
\end{tabular}

TABLE IV-Detection of adenoma in screening programme by Hemoccult II and immunological techniques. Values are proportions (percentages)

\begin{tabular}{|c|c|c|c|}
\hline & \multicolumn{2}{|c|}{ Hemoccult II } & \multirow{2}{*}{$\begin{array}{c}\text { Immunological } \\
\text { technique }\end{array}$} \\
\hline & Not rehydrated & Rehydrated & \\
\hline Subjects & $14 / 40(35)$ & $22 / 40(55)$ & $34 / 40(85)$ \\
\hline Samples & $32 / 238(13)$ & $74 / 238(31)$ & $87 / 237(37)$ \\
\hline
\end{tabular}

TABLE V-False positive results detected in screening programme by Hemoccult II and immunological techniques. Values are proportions (percentages)

\begin{tabular}{lccc}
\hline & \multicolumn{2}{c}{ Hemoccult II } & \\
\cline { 2 - 3 } & Not rehydrated & Rehydrated & $\begin{array}{c}\text { Immunological } \\
\text { technique }\end{array}$ \\
\hline Subjects & $28 / 1304(2)$ & $55 / 1302(4)$ & $50 / 1304(4)$ \\
Samples & $58 / 7824(<1)$ & $155 / 7812(2)$ & $109 / 7824(1)$ \\
\hline
\end{tabular}

TABLE VI-Findings in subjects with different numbers of positive results by Hemoccult II and immunological techniques. Values are numbers of subjects

\begin{tabular}{|c|c|c|c|c|c|c|}
\hline \multirow[b]{2}{*}{ Finding } & \multicolumn{6}{|c|}{ No of positive samples (out of six) } \\
\hline & 1 & 2 & 3 & 4 & 5 & 6 \\
\hline \multicolumn{7}{|c|}{ Hemoccult II not rehydrated } \\
\hline Carcinoma & & 2 & 2 & 3 & & 4 \\
\hline Adenoma & 7 & 2 & 2 & & & 2 \\
\hline False positive result & 11 & 11 & & 5 & 1 & \\
\hline \multicolumn{7}{|c|}{ Hemoccult II rehydrated } \\
\hline Carcinoma & 2 & 2 & 1 & 1 & 2 & 7 \\
\hline Adenoma & 4 & 5 & 2 & 4 & 2 & 4 \\
\hline False positive result & 11 & 20 & 4 & 12 & 4 & 4 \\
\hline \multicolumn{7}{|c|}{ Radial immunodiffusion } \\
\hline Carcinoma & 2 & 3 & 1 & 3 & 4 & 6 \\
\hline Adenoma & 15 & 4 & 3 & 6 & 2 & 3 \\
\hline False positive result & 27 & 7 & 6 & 2 & 6 & 2 \\
\hline
\end{tabular}

with the unrehydrated Hemoccult II technique was greater than that with the rehydrated technique and also the immunological technique. As expected, the yield of both cancers and adenomas increased with each decade of age between 50 and 79 years.

\section{Discussion}

In our screening programme $1.4 \%$ of all subjects participating had colorectal carcinoma and $4 \cdot 4 \%$ had either carcinoma or adenomas, values that are far higher than those observed by other groups. ${ }^{10}$ This may be because the immunological technique is much more sensitive than Hemoccult II, which has been used in screening programmes till now. Other factors such as more complete follow up investigations, older subjects, and more subjects with a family history of bowel polyps or carcinoma may, however, have contributed.

Colonoscopy has been shown to be better than a barium enema in diagnosing colorectal neoplasms, ${ }^{112}$ and its routine use may have contributed to the many carcinomas diagnosed. The incidence of carcinoma increases with age, and thus screening an older population will detect more carcinomas. The mean age of subjects who participated, however, was $58.5(12.5)$ years compared with the mean age of incidence of $67 \cdot 2$ years in New South Wales in 1982.13 Thus the age of subjects in this study probably did not contribute to the many carcinomas detected. Most of the other studies did not give a mean value for the age of the populations screened or the proportion with a family history of bowel cancer or polyps.

The large percentage of carcinomas found may have been biased by the fact that our subjects selected themselves and participated because first degree relatives had the disease; such subjects would have about a threefold risk of developing carcinoma. In our study 20 out of $136(15 \%)$ subjects with positive stools who were investigated had first degree relatives with carcinoma. Winawer et al found this value to be $16 \%$ in their screened subjects. ${ }^{14}$ Another study found 
that 36 out of $328(11 \%)$ of randomly selected men (mean age 66 $(4 \cdot 7)$ years) had a first degree relative with colorectal cancer. ${ }^{15}$ The data therefore suggest that the population in our study was "enriched" to only a small extent with subjects who had a family history of carcinoma or adenoma of the bowel and that this effect was not the main reason for the high prevalence of carcinomas.

Radial immunodiffusion detected bleeding in all of the carcinomas found but not necessarily all of those present in the population screened as subjects with negative results were not investigated. In this study Hemoccult II with and without rehydration detected mainly the more advanced carcinomas and only $64 \%$ and $55 \%$, respectively, of those subjects with stage A carcinomas, whereas radial immunodiffusion additionally detected all of the stage $A$ carcinomas. Early diagnosis and removal of stage A carcinomas is potentially of much greater benefit for survival than detection of the later stages, C and D, and a higher rate of survival would be expected for subjects screened with the immunological rather than the Hemoccult II technique. In a previous study when 40 patients who presented with clinical colorectal carcinoma were tested with radial immunodiffusion and Hemoccult II with and without rehydration $100 \%, 88 \%$, and $72 \%$ of patients, respectively, were found to have positive stools. ${ }^{5}$ The inclusion of patients with visible blood in that and similar studies resulted in a high detection rate for Hemoccult II than in the present screening programme. The proportion of stage A carcinomas detected $(58 \%)$ in the screening programme was much higher than that found in patients presenting clinically (15-25\%), and conversely the proportion of patients with stage D carcinomas in our screened patients was much lower.

The shift to earlier pathological staging of tumours detected by screening is similar to that found in other screening programmes ${ }^{10}$ and suggests that screened populations may have a lower mortality from bowel cancer than unscreened populations. The degree of such benefit will have to await the results of controlled trials, ${ }^{141617}$ but calculations based on the proportions of different Dukes's stages of the cancers in screened and unscreened populations and the five year survival of patients at the different stages suggest that screening may raise five year survival from about $42 \%$ to about $70 \%$ if lead time and length biases are not large. (Screening programmes for cancers can give misleadingly optimistic estimates of improved survival because of two different forms of bias in the mortality data known as lead times and length biases. Lead times occur because tumours are diagnosed before they would have been diagnosed from symptoms, and this, in the absence of effective treatment, may give a seemingly increased length of survival from the time of diagnosis that is is in fact due only to an increased time of observation. Length biases occur because more slowly growing tumours exist in the screened population for longer, thereby increasir $g$ their chances of being detected by screening techniques; it is * ought that slowly growing tumours, even in the absence of effc tive treatment, are associated with longer survival.) The prog-ession of the mean ages-58.5 (12.5) years in the overall pop' dtion screened, 61.5 $(11.4)$ years in those with adenomas, and $6.5(9 \cdot 3)$ years in those with carcinomas-fits with the hypothes:; that adenomas may progress to carcinomas.

We could not assess whether all of the carcinomas present in the population studied were detected by radial immunodiffusion without investigating all 1328 subjects by colonoscopy. This would have been impractical, time consuming, costly, and probably unethical and unacceptable to many subjects without symptoms who had negative results on testing. In addition, no screening programme can have a higher sensitivity for a lesion than that of the follow up investigation. Although colonoscopy is better than barium enema in detecting carcinomas, its rate of detection is probably not $100 \%$, especially for carcinomas in small polyps.

The detection of non-malignant polyps in the screened population was also increased with radial immunodiffusion compared with the chemical technique. The 40 subjects with adenomas identified by the programme had these removed at colonoscopy, thereby eliminating the chance of their developing into cancer. The characteristics of the polyps removed in this screening programme will be reported separately. Evidence suggests, however, that removal of colorectal polyps results in fewer colorectal carcinomas developing, ${ }^{18}{ }^{19}$ which implies that the screening programme will have a second effect in reducing mortality from colorectal cancer in the screened population. We do not, however, have enough data to estimate the size of this benefit.

The immunological method used in our screening programme has advantages over other techniques for detecting occult blood in that it is specific for human haemoglobin, the haemoglobin remains stable for several weeks, ${ }^{6}$ and it is much more sensitive to bleeding from the colon than from the upper gut (unpublished data). As carcinomas bleed intermittently obtaining specimens over six days noticeably reduces the number of false negative results. Our data showed that if two or more specimens were positive by the immunological technique the rate was $60 \%$ that either a carcinoma or an adenoma would be found compared with $39 \%$ if only one specimen was positive.

The method used in screening programmes should have not only a high degree of sensitivity but also a high specificity with an acceptably low rate of false positive results. Our study showed that for the immunological technique a balance has been struck between the higher sensitivity on the one hand and the higher rate of false positive results with lower specificity on the other. The opposite is true with the Hemoccult II technique without rehydration. The Hemoccult II rehydrated technique has a sensitivity, specificity, and rate of false positive results that are worse than those of the immunological technique. Winawer et al abandoned the use of the rehydrated technique because of the noticeable increase in the rate of false positive results. ${ }^{14}$

We think that a sensitivity of about $50 \%$ for the Hemoccult II without rehydration is no longer acceptable and that a rate of false positive results of $4 \%$ for the immunological technique is low enough to enable a screening programme for bowel cancer to proceed without investigatory facilities being overwhelmed or excessive costs being incurred. Costs and the requirements for colonoscopy may be reduced by a variety of strategies - for example, screening only subjects older than 50 or using fibreoptic sigmoidoscopy and double contrast barium enema instead of colonoscopy. Whichever strategies for screening are adopted our study suggests that immunological techniques for detecting faecal occult blood detect more carcinomas and adenomas than chemical techniques and should be used in future screening programmes.

\section{Re1 :rences}

1 Buder JP. Screening for colorectal cancer. Dig Dis Sci 1986;31(suppl 9):43-56S

2 Macrae FA, St John DJB, Calagiore P, Taylor LS, Legge WJ. Optimum dietary conditions for Hemoccult testing. Gastroenterology 1982;82:899-903.

3 Doran J, Hardcastle JD. Bleeding patterns in colorectal cancer: the effect of aspirin and the implications for faecal occult blood testing. Brf Surg 1982;69:711-3.

4 Macrae FA, St John DJB. Relationship between patterns of bleeding and Hemoccult sensitivity in patients with colorectal cancers or adenomas. Gastroenterology 1982;82:891-8.

5 Kapparis A, Frommer DJ. Immunological detection of occult blood in bowel cancer patients. Br $\mathcal{F}$ Cancer 1985;52:857-61.

6 Frommer DJ, Kapparis A. Faecal occult blood testing. Lancet 1983;ii:738.

7 Frommer DJ, Logue T. Comparison of five guaiac resin paper tests for demonstrating the presence of blood in faeces. Aust NZF Med 1981;11:494-6.

8 Songster CL, Burrows GH, Jarrett DD. Immunochemical detection of fecal occult blood-the fecal smear punch-disc test: a new non-invasive screening test for colorectal cancer. Cancer 1980;45:1099-102.

9 Schatzkin A, Connor JR, Taylor PR, Bunnag B. Comparing new and old screening tests when a reference procedure cannot be performed on all screenees-example of automated cytometry for early detection of cervical cancer. Am $\mathcal{F}$ Epidemiol 1987;125:672-8.

10 Simon JB. Occult blood screening for colorectal carcinoma: a critical review. Gastroenterology 1985;88:820-37

11 Hunt RH. Towards safer colonoscopy. Gut 1983;24:371-5

12 Boulos PB, Karamanolis DG, Salmon PR, Clark CG. Is colonoscopy necessary in diverticular disease? Lancet 1984; i:95-6.

13 New South Wales Central Cancer Registry. Cancer in New South Wales, incidence and mortality, 1982. Sydney: New South Wales Central Cancer Registry.

14 Winawer SJ, Andrews M, Flehinger B, Sherlock P, Schottenfeld D, Miller DG. Progress report on controlled trial of fecal occult blood testing tor the detection of colorectal neoplasia. Cancer on controlled trial

15 Chapius PH, Goulston KJ, Dent OF, Tait AD. Predictive value of rectal bleeding in screening for rectal and sigmoid polyps. Br Med f 1985;290:1546-8

16 Gilbertsen VA, McHugh RB, Schuman LM, Williams SE. The earlier detection of colorectal cancers. A preliminary report of the results of the occult blood study. Cancer 1980;45:2899-901.

17 Hardcastle JD, Farrands PA, Balfour TW, Chamberlain J, Amar SS, Sheldon MG. Controlled trial of faecal occult blood testing in the detection of colorectal cancer. Lancet 1983;ii:1-4.

8 Hertz RE, Deddish MR, Day E. Value of periodic examinations in detecting cancer of the rectum and colon. Postgrad Med 1960;27:290-4.

19 Gilbertsen VA. Proctosigmoidoscopy and polypectomy in reducing the incidence of rectal cancer. Cancer 1974;34:936-9.

(Accepted 23 December 1987) 